\title{
REPERTORIO BIBLIOGRÁFICO SOBRE EL RÉGIMEN
} POLÍTICO VENEZOLANO

\author{
CAYETANO NÚÑEZ RIVERO \\ Profesor Titular de Derecho Político \\ UNED
}


1. Eeconomia y Sociedad.-1.1. Aspectos económicos. 1.2. Aspectos sociales. 2. Historia Politica.-3. Constitución.-4. ProCESOS ElECTORALES. - 5. Fuerzas POLITICAS Y SOCIALES. - 5.1. Partidos políticos. Obras generales. 5.2. Acción Democrática. 5.3. C.O.P.E.I. (Social Cristianos). 5.4. Partido Comunista de Venezuela. 5.5. M.A.S. 5.6. Otras fuerzas políticas. 5.7. Sindicatos y organizaciones obreras. 5.8. Fuerzas Armadas (incluye movimientos guerrilleros). 
Revista de Derecho Político, núm. 34, 1991, pp. 387-429

\title{
REPERTORIO BIBLIOGRÁFICO SOBRE EL RÉGIMEN POLÍTICO VENEZOLANO *
}

\author{
POR \\ CAYETANO NÚÑEZ RIVERO \\ Profesor Titular de Derecho Político \\ UNED
}

1. ECONOMIA Y SOCIEDAD

\subsection{Aspectos económicos}

Álvarez, Mercedes M.: Comercio, comerciantes y sus proyecciones en la independencia de Venezuela. Ed. Tipográfica Vargas. Caracas 1963.

APONTE, Carlos: «Posiciones empresariales venezolanas ante el Pacto Andino", en Cuadernos del Cende, n. ${ }^{\circ}$ 4. Caracas 1984.

ARMADA, Sergio: «El comportamiento de la economía venezolana en la actual crisis mundial», en Cuadernos del Cende, n. ${ }^{\circ}$. Caracas 1983.

-: «La crisis del modelo de desarrollo venezolano ¿crisis de estrategia o política errónea?», en Economía y Desarrollo, n. ${ }^{\circ} 11$. Quito 1986.

—: La economia venezolana. Ed. Siglo XXI. Bogotá 1977.

ARAúJo, O.: «Caracterización histórica de la industrialización en Venezuela», en Economia y Ciencias Sociales, n. ${ }^{\circ}$ 4. Caracas 1964.

* Dedicado a la memoria del Dr. ORLANDO TOVAR TAMAYO. 
ARCILla Farias, Eduardo: Economía colonial de Venezuela. Ed. Italgráfica. Caracas 1973.

-: El Real Consulado de Caracas. Ed. Edime. Caracas 1957.

-: El siglo ilustrado en América. Reformas económicas del siglo. Edita Ministerio de Educación. Caracas 1955.

-: Historia del monopolio. El estanco del tabaco en Venezuela. Ed. F.H.C. Universidad Central de Venezuela. Caracas 1977.

Avalos, I., y Anoronsi, M.: La Planificación. Ed. Ateneo. Caracas 1983.

Banco Industrial de Venezuela: El desarrollo de Venezuela. Situación actual y perspectiva. Madrid 1981.

BAPTISTA, Asdrúbal: La encrucijada de la economía venezolana. Ed. IESA. Caracas 1987.

BAPTISTA, A., y Mommer, B.: El petróleo en el pensamiento económico venezolano. Ed. IESA. Caracas 1987.

Betancourt, Rómulo: Venezuela: política y petróleo. Ed. Senderos. Bogotá 1969.

-: Venezuela, dueña de su petróleo. Ed. Centauro. Caracas 1975.

BIGLER, G.: La política y el capitalismo de Estado en Venezuela. Ed. Tecnos. Madrid 1981.

Bitar, S., y Truncoso, E.: El desafío industrial de Venezuela. Ed. Pomaire. Argentina 1983.

BLANK, David: Venezuela: Politics in a Petroleum Republic. Praeger. New York 1984.

BouRguignon, François: Venezuela le clase petrolière, 1973-85, en Problèmes d'Amérique Latine, n. $^{\circ} 75$. Paris, 1985.

Brito Figueroa: El cuadro histórico de la propiedad territorial en las colonias hispanoamericanas. Fondo Editorial C. de Fuenmayor. Caracas 1982. 
Brito Figueroa: Historia Económica y Social de Venezuela. Estructura para su estudio. Ed. Universidad Central de Venezuela. Caracas 1979.

-: "Historia Sociológica, agraria y rural de Venezuela», en Revista Universitaria de Ciencias del Hombre, $\mathrm{n}^{\circ}{ }^{3}$. Ed. Universidad José

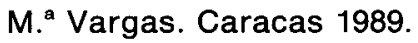

-: La estructura económica de Venezuela colonial. Ed. Universidad Central de Venezuela. Caracas 1978.

Caldera, Rafael: La nacionalización del petróleo. Nueva Política. Venezuela 1975.

Cardoso, C., y Pérez Brignoli, H.: Historia Económica de América Latina. Ed. Grijalbo, Barcelona 1984.

Carrera Damas, G.: Boves, aspectos socioeconómicos de su acción histórica. Caracas 1968.

Carrillo Batalla, T.: Historia de las finanzas públicas en Venezuela (10 vols.). Caracas 1969-73.

Carvallo, G., y Rios de Hernández, J.: Temas de la Venezuela Agroexportadora. Fondo Editorial Tropykos. Caracas 1984.

CASTILLO, Ocarina: Agricultura y política en Venezuela: 1948-1958. Ed. Univ. Central de Venezuela. Caracas 1985.

CORdova, Armando: Inversiones extranjeras y subdesarrollo. Ed. FACES. Univ. Central Venezuela. Caracas 1979.

Córdova Cañas, V.: Capitalismo, subdesarrollo y modo de vida en Venezuela. Ed. Univ. Central de Venezuela. Caracas 1980.

CORONEL, G.: The nationalization of the Venezuelan oil industry: from technocratic succes to political failvre. Lexington Books. Massachusetts 1983.

Cunill Grau, Pedro: La diversidad territorial, base del desarrollo venezoIano. Ed. Cuadernos Lagoven. Caracas 1981.

-: Recursos y territorios en la Venezuela posible. Ed. C. Lagoven. Caracas 1985. 
Dos SANTOS, Theodomio: La crisis norteamericana y América Latina. FACES. Univ. Central de Venezuela, Caracas 1974.

Escobar, Gustavo: El laberinto de la economia. (En el caso Venezuela, una ilusión de armonía). Ed. IESA. Caracas 1986.

-: La productividad en la industria venezolana. Ed. Consejo Nacional de Recursos Humanos. Caracas 1981.

FAJARDO CORTEZ, Victor: "La crisis económica y el proceso de ajuste en 1983", en Cuadernos del Cendes, n. ${ }^{\circ} 6$. Caracas 1986.

Franco-Garcia, José M. ${ }^{\text {a. }}$ El Derecho y la Reforma Agraria. Univ. de los Andes. Mérida 1981.

Garaicoechea, M. F.: El comercio exterior y la estrategia del desarrollo económico de Venezuela. Caracas 1969. •

GonZALEZ, David (Comp.): El nuevo orden económico internacional. Un reto para el socialismo democrático. ILDIS. Caracas 1981.

Gonzalez García-Baquero, A.: Comercial colonial y guerras revolucionarias. Ed. Esc. Estudios hispanoamericanos de Sevilla. Sevilla (España) 1972.

GrifFIN, C.: «Economic and Social Aspects in the era of Spanish American Independence", en Hispanic America, Historica Review, 29. 1949. .

Harwich Valenilla, N.: "La crisis de 1929 en América Latina. El caso de Venezuela», en Lecciones Magistrales, n. ${ }^{\circ}$ 2. U.S.M. Caracas 1984.

Hausmann, R., y Maraver, G.: «La crisis económica de Venezuela», en Cuadernos del Cendes, vol. 1, n. ${ }^{\circ} 1,1983$.

IZARD, M., y otros: Politica y Economía en Venezuela, 1810-1976. Caracas 1976.

KARL, Terry Lynn: «Petroleum and political pacts: the transition to democracy in Venezuela", en Latin America Research Review. vol. 22.1. 1987.

KrIvor, Ruth de: «Venezuela ante el proceso de integración andino». Integ. Latinoamericana, n. ${ }^{\circ}$ 127. Buenos Aires 1987. 
LANZA, E.: El Sub-imperialismo venezolano. Ed. C. Apunte. Caracas 1980.

Lombardi, John: Venezuela. La búsqueda del orden. El sueño del progreso. Ed. Crítica. Barcelona 1985.

LoSADA, Benito R.: La transición económica en tránsito de la Dictadura a la Democracia en Venezuela. Ed. Salcedo, 1978.

LUCEnA Salmoral, M.: La economía americana del primer cuarto del siglo XIX. Ed. Academia Nacional de la Historia. Caracas 1983.

LUZARdo, R.: Notas histórico-económicas, 1928-1963. Ed. Sucre. Caracas 1963.

Machado Acedo, C., y Padrón Quero: Estado y grupos económicos en Venezuela. Ed. Ateneo. Caracas 1981.

-: La diplomacia de López Contreras y el tratado de reciprocidad comercial con Estados Unidos. Ed. Instituto de Asuntos Internacionales. Caracas 1987.

Malabe MATA, Héctor: Formación histórica del antidesarrollo de Venezuela. Ed. Oveja Negra. Bogotá 1980.

MARTINEZ, R., y otros: El desarrollo de Venezuela: situación actual y perspectiva. Banco Industrial de Venezuela, 1981.

MARTINez de Delgado, E.: «La región Carúparo y las repercusiones de la crisis de 1929", en Revista Universitaria de Ciencias del Hombre, n. ${ }^{\circ}$ 3. Caracas 1989.

Mezger, D.: Petróleo y ecodesarrollo en Venezuela. ILDIS. Caracas 1981.

MOMMER, Bernard: «La renta petrolera, su distribución y cuentas nacionales. El ejemplo de Venezuela", en Cuadernos del Cendes, n. ${ }^{\circ}$ 5. 1986.

-: «Los precios del petróleo ¿economía o política?», en Planificación y política, n. ${ }^{\circ}$ 1. Instituto Venezolano de Planificación. Caracas 1983.

MOMMER, B., y BAPTISTA, A.: El petróleo en el pensamiento económico venezolano. Ed. IESA. Caracas 1987.

Osorıo, Alejandro: La economía del cacao en Venezuela. MAC. Caracas 1936. 
Pazos, I.: Política de desarrollo económico: colección de documentos, de artículos, ponencias e informes, 1949-1984. Banco Central de Venezuela. Caracas 1985.

Pérez VILA, M., y otros: Política y Economia en Venezuela (1810-1976). Ed. Fundación John Boulton. Caracas 1976.

Polanco Martinez, T.: Esbozo sobre Historia Económica Venezolana, 14981810. Ed. Ancota. Caracas 1980.

Purroy, M. T.: Estado e industrialización en Venezuela. Ed. Hermanos Vadell. Valencia 1982.

RABE, Stephan G.: The road to OPEC United States relations with Venezuela, 1919-1976. Univ. of Texas. Press, Austin 1982.

RANGel, D. A.: Capital y desarrollo: la Venezuela agraria. Faces. Univ. Central de Venezuela. Caracas 1983.

ReBolledo, G.: Un modelo estructural de la inflación: el caso venezolano. Univ. Central de Venezuela. Caracas 1983.

RIos, Josefina: La Hacienda Venezolana. Ed. Tropykos. Caracas 1988.

RodRIGUEZ, Gumersindo: El nuevo modelo de desarrollo venezolano. Ed. Grupoconsult. Caracas 1979.

-: ¿Era posible la Gran Venezuela? Ed. Ateneo. Caracas 1986.

Rodriguez Campos, Manuel: Venezuela, 1948-88. El proceso económico y social y la dictadura. Alianza Crítica Editorial. Caracas 1983.

Rosales, M. T., y Fontana, H.: El desafio agrícola. Políticas para la agricultura venezolana en los años 80. Edicampa. Caracas 1983.

Salazar, José: "Notas sobre la economia de Carúparo a fines del siglo XIX", en Revista Tierra Firme, n. ${ }^{\circ} 3$. Caracas 1983.

Serulle, J., y Boin, J.: Fondo Monetario Internacional. Deuda Externa y crisis mundial. MEPALA. Madrid 1984.

Sequera de VerginI, I.: Visión Geopolítica de Venezuela. Ed. Salvat. Caracas 1986. 
Silva Michelena, Héctor: «Proceso y crisis de la economía nacional, 196073", en Nueva Ciencia, n. ${ }^{\circ} 1.1974$.

Silva Michelena, J. A., y Sountag Heinz: «Una estrategia para el desarrollo», en Metas, n. ${ }^{\circ}$ 12. 1983.

Sountag Heinz; R.: «Entorno al Estado productor: el caso de Venezuela», en Pensamiento Iberoamericano, n. ${ }^{\circ} 5.1984$.

Sountag Heinz, R., y de La Cruz, R.: «Estado e industrialización en Venezuela", en Nova América, n. ${ }^{\circ}$ 5. 1982.

TANDRÓn, Humberto: El Real Consulado de Caracas y el comercio exterior de Venezuela. Ed. FHE. Universidad Central de Venezuela. Caracas 1976.

TovaR, Ramón: Imagen geoeconómica de Venezuela. Ed. Vadell Hermanos. Valencia 1985.

一: «Trabajo, Desarrollo y Organización Social», en Revista Universitaria de Ciencias del Hombre, n. ${ }^{\circ}$. Universidad José M. ${ }^{a}$ Vargas. Caracas 1989.

Tugwell, Franklin: Petroleum Policy and the Political Process en Venezuela: The Democratic Experience. Ed. Martz y Myers, 1977.

-: The politics of oil in Venezuela. Stanford. Univ. Press. Stanford. California 1975.

UrRIZA, Manuel: El empresario venezolano y el Pacto Andino. Instituto de Altos Estudios de América Latina. Caracas 1984.

VACCHINO, Juan M.: Integraciones económicas regionales. Univ. Central de Venezuela. Caracas 1981.

VAlLenilla, L.: Auge, declinación y porvenir del petróleo venezolano. Caracas 1973 .

YÁ̃̃ez BetancouRT, L.: «La economía venezolana: problemas y perspectivas", en Trimestre Económico, n. ${ }^{\circ}$ 216. México 1987.

Zambrano, L., y otros: «La deuda externa de Venezuela: situación, perspectivas e implicaciones", en Resumen, 1983. 


\subsection{Aspectos sociales}

ARANDA, Sergio: Las clases sociales y el Estado en Venezuela. Univ. Central de Venezuela. Caracas 1969.

Arango, A.; Lima, L.; Argüello Dumas, y otros: La mujer inmigrante. ILDIS. Caracas 1986.

ARAúJo, Orlando: Venezuela violenta. Espendos. Caracas 1968.

Bidegain, G., y otros: Estado actual de los estados de población en Venezuela. ILDIS. UCAB. Caracas 1987.

—: Las migraciones laborales colombo-venezolanas. ILDIS. UCAB. Nueva Sociedad. Caracas 1987.

Blanco, A. E.: Cuba, patria del exilio venezolano. Ed. Centauro. Caracas 1982.

Blumenthal, Hans.; Rey, Juan Carlos, y otros: Venezuela hacia el año 2000. Desafios y opciones. Ed. Nueva Sociedad. Caracas 1987.

BonilLA, Frank: El fracaso de las élites. Caracas 1972.

Brito, B.: Los medios de comunicación social en Venezuela. C. Gumulla. Caracas 1984.

Cardoso Galve, German, y otros: La Región histórica. Fondo Editorial Tropykos, 1988.

CARIAS, Rafael: ¿Quiénes somos los venezolanos? Antropología cultural del venezolano. Ed. ISSFE. Les Teques, 1982.

Carrera Damas, Germán: «A propósito de la hipótesis de Charles G. Griffin. Cuestiones económico-sociales de la emancipación", en Cuestiones de historiografía venezolana. Eds. Avance, $\mathrm{n}^{\circ}$ 7. Univ. Central de Venezuela. Caracas 1974.

-: Una nación llamada Venezuela. Proceso socio-histórico de Venezuela (1810-1974). Ed. Dirección de Cultura. Univ. Central de Venezuela. Caracas 1980. 
CaRRera DAMAS, Germán: Venezuela: proyecto nacional y poder social. Ed. Crítica. Barcelona 1986.

ChACON, Alfredo: Cultura y Dependencia. Ed. Monte Ávila. Caracas 1975.

Chossudovsky, M.: La miseria en Venezuela: mapa de la pobreza en Venezuela. Talleres Gráficos. Barcelona 1982.

Dél Corro, Alejandro: Venezuela y la violencia. CIDOC. Cuernavaca 1968.

-: Violencia, 1960-68. CIDOC. Cuernavaca 1968.

EscobAR, G.: La estructura de sueldos y salarios en Venezuela. Ed. Consejo Nacional de Recursos Humanos. Caracas 1982.

Escovar Salom, R.: Apertura hacia el futuro en Venezuela. Ed. Legoven. Caracas 1985.

FernAndez, L. M.: El Venezolano. Ed. Universidad Nacional de Zulia. Maracaibo.

GIL YePes, José Antonio: El reto de las élites. Ed. Tecnos. Madrid 1978.

Gómez, A., y DIAZ, L. M.: La moderna esclavitud. Los indocumentados en Venezuela. Ed. Oveja Negra. Bogotá 1983.

Hernandez Vera, R.: Pasos y huellas. Personajes y acontecimientos venezolanos. Corporación cientifica. Madrid 1985.

Humdert, Jules: Los origenes venezolanos. Ed. Academia Nacional de la Historia. Caracas 1976.

IZARD, M.: Series estadisticas para la historia de Venezuela. Mérida 1970.

LAMBI INSÚA, Luis: «El grupo Acanguas: surgimiento y consolidación de una burguesía regional con base agrícola», en Cuadernos del Cendes, n. ${ }^{\circ}$. Caracas.

LERMEN, Elisa: «Venezolanos de hoy en día: del silencio posgomocista al ruido mayamero", en El Caso Venezuela. Ed. IESA. Caracas 1986.

LieVwen, E.: Venezuela. Ed. Sudamericana. Buenos Aires 1964. 
Lombardi, J. V., y otros: Venezuela. New York 1982.

LÓPEZ CONTRERAS, Eleazar: Proceso político-social, 1928-1936. Sucre. Caracas 1963.

Morales Álvarez, Juan: Los extranjeros con carta de naturaleza en las Indias durante la segunda mitad del siglo XVIII. Eds. Academia Nacional de la Historia. Caracas 1980.

Oficina de Educación Iberoamericana: Venezuela, series estadísticas. 4. volúmenes. Madrid.

Perdomo, Rp.: “Corruption and Bussiness in present day Venezuela», en Journal of Bussiness etnics, 1990 . Vol. 9, n. ${ }^{\circ} 7$.

Petkoff, T.: «Venezuela en el mundo. Seguridad Nacional desde la perspectiva del cambio social», en Seguridad, Defensa y Democracia. Ed. Pu Aníbal Romero, 1980.

Petzoldt, F., y BeVILACQua: Nosotros también nos jugamos la vida. Testimonios de la mujer venezolana en la lucha clandestina, 1948-1958. Ed. Ateneo. Caracas 1979.

Quintero, Rodolfo: Caminos para nuestros pueblos. Univ. Central de Venezuela. Caracas 1969.

—: La cultura del petróleo. Univ. Central de Venezuela. Caracas 1975.

Rangel, D.: La oligarquía del dinero. Ed. Fuentes. Caracas 1982.

Rangel Belis, Gonzalo: Sector económico, industrialización y conflictos laborales en Venezuela. Ed. Hermanos Vadell. Valencia 1985.

Silva MichelenA, José A. (Coordinador): Venezuela hacia el 2000. Desafios y Opciones. Ed. Nueva Sociedad. Caracas 1987.

Siso, Carlos: La formación del pueblo venezolano. Ed. Es. Siso. Madrid 1982.

Sgambati, Sonia: La mujer ciudadana de segundo orden. Fondo Editorial Común. Caracas 1975.

TANNEN, Chris: Venezuelan migrations. An analysis of problems migration. Ed. Verlang Ruegger. 1980. 
Travieso, Fernando: Ciudad, Región y Subdesarrollo. Fondo Editorial Común. Caracas 1973.

Troconis de Veraloecueta, E.: El proceso de la inmigración en Venezuela. Ed. Academia Nacional de la Historia. Caracas 1986.

ULLRICH, Detlev: Bariopolitik en Caracas. Eine socialem-pirische untasuchaug an Beispiel der Erwachsenenbilddung und der Stadtteilabeit. Breitenbach - Saabrücker 1984.

Velde de Ramírez, I., y otros: La corrupción en Venezuela. Ed. Hermanos Vadell. Valencia 1986.

\section{HISTORIA POLITICA}

ACAdemia Nacional de la Historia: El movimiento emancipador en Hispanoamérica. Ed. Academia Nacional de la Historia. Caracas 1961.

Aguado, P.: Historia de Venezuela. 2 vols. Ed. Atlas. Madrid 1980.

Alvarado Migeot, Judith: "La enseñanza de la historia política en Venezuela», en Revista Universitaria de Ciencias del Hombre, n. ${ }^{\circ} 3$. Univ. José García Vargas, 1989.

Arcila larias, Eduardo: El siglo ilustrado en América. Ed. Ministerio Educación y Ciencia. Caracas 1957.

Arellano Moreno, A.: Guía de Historia de Venezuela, 1492-1945. Ed. Edime. Caracas 1958.

-: Miradas de la historia política de Venezuela. Imp. Nacional. Caracas 1967.

BARALt, R. M., y DIAZ, R.: Resumen de la historia de Venezuela desde el año 1797 hasta el de 1850. 2 vols. Bruges 1939.

Bello, Andrés: Antología de discursos y escritos. Editora Nacional. Madrid 1976. 
BeLmonte GuerRero, A.: Ensayo sobre la historia política de Venezuela, 1917-1968. Academia Nacional de la Historia. Caracas 1981.

Brito Figueroa, F.: Venezuela, siglo $X X$, Casa de las Américas. Las Habana.

Carpio Castillo, Rubén: Geopolítica de Venezuela. Ed. Ariel, Seix Barral. Caracas 1981.

CANTER, Juan: La logia Lautaro y la independencia de América. Ed. Crítica Histórica. Buenos Aires 1983.

Carnicelli, Américo: La Masonería en la independencia de América, 18101830. Secretos de la Historia. Bogotá 1970.

Carrera, G.: Historia de historiografía venezolana. Universidad Central de Venezuela. Caracas 1961.

Castellón, Hello: Guía histórica de la Masonería venezolana. Ed. Lito Jet. Caracas 1985.

Cayama Martinez, Rafael: «De Rescio a Bolívar», en Epistolario de la Primera República. Sesquicentenario de la Independencia. Tomo II. Biblioteca de la Academia Nacional. Caracas 1960.

Сово, Lourdes: Estudio de las relaciones internacionales en Venezuela. Ed. Ministerio de Relaciones Exteriores. Caracas 1982.

Chávez, L. F., y Vivas, L.: Venezuela. Ed. Panam. México 1968.

Febres Cordero, Julio: Historia del periodismo y de la imprenta en Venezuela. Academia Nacional de la Historia. Col. Fuentes, n. ${ }^{\circ} 158 . \mathrm{Ca}-$ racas 1983.

Figuera, Guillermo: La Iglesia y su doctrina en la Independencia de América. Sesquicentenario de la Independencia. BD. Academia Nacional de la Historia. Caracas 1960.

FuenMAYOR, Juan Bautista: Historia de la Venezuela politica contemporánea, 1899-1969. Caracas 1978.

—: 1928-1948. Veinte años de política contemporánea. Eds. Mediterráneo. Madrid 1968. 
Fundación Polan: Diccionario de historia de Venezuela. 3 vols. Caracas 1989.

GaRcia Chuecas, Héctor: Documentos relativos a la revolución de Gual y España. Instituto Panamericano de Geografia e Historia. Caracas 1949.

—: Siglo XVIII venezolano. Ed. Edime. Caracas-Madrid, s/f.

GonzÁLEZ, Eloy G.: Historia de Venezuela. 2. vols. Ed. Elite. Caracas 1930.

GonzÁlez Guinan, T.: Historia contemporánea de Venezuela. 15 vols. Caracas 1954.

Gosselman, C. A.: Informes sobre los Estados Sudamericanos en los años de 1837 a 1838. Estocolmo 1962.

Grases, P., y Pérez VILA, M. (Comp.) (incluye texto de Rómulo Betancourt): La Independencia (1810-1830). 5 vols. Eds. Presidencia de la República. Caracas 1963.

Instituto de Estudios Politicos: La agenda de la política exterior de Venezuela. Caracas 1983.

IZARD, M.: La lucha por la libertad en Venezuela, 1777-1830. Ed. Tecnos. Madrid 1977.

—: El miedo a la revolución. Ed. Tecnos. Madrid 1979.

Kaldone G. NuelHed: «Bolívar y el Tercer Mundo», en Anuario de Estudios Bolivarianos, n. ${ }^{\circ}$, año 1. Univ. Simón Bolívar, 1990.

LecunA, V.: La revolución de Queipa. Caracas 1954.

LEVINE, Daniel: Religion and politicals in Latin America, The Catholic Church in Venezuela and Colombia. Princeton. Univ. Press. 1981.

LIENDO Gomez, C. B.: «La masonería en Venezuela. Influencia en la Emancipación", en Anuario de Estudios Bolivianos, n. ${ }^{\circ}$. Univ. Simón Bolivar, 1990.

LOMBARDI, J. V., y otros: Venezuela history. Boston. Mass. 1977. 
Magallanes, M. V.: Historia política de Venezuela. Ed. Monte Ávila. Caracas 1975.

Martínez Sanchez, A.: Nuestras contiendas civiles. Caracas 1949.

Maza Zavala, D. F.: Historia de medio siglo en Venezuela, 1926-1975. UNAM. Siglo XXI. México 1977.

Medina RuBio, Arístides: Metrología histórica e imperio regional. Ruta. Tierra Firme. Caracas 1968.

MiJARES, Augusto: «La evolución política, 1810-1960», en Venezuela Independiente, 1810-1960. Fundación Eugenio Mendoza. Caracas 1962.

MORón, Guillermo: Breve historia de Venezuela. Eds. Austral. Madrid 1979.

Morón, G., y otros: Historia de Venezuela. 5. vols. Italgráfica editores. Caracas 1971.

MORÓN; MoIsÉs, y otros: Apuntes teóricos y técnicos para la construcción de la historia local y regional. Fondo Ed. Tropykos. Caracas 1983.

NaIM, M.; PIñango, R., y otros: El caso Venezuela. Una ilusión de armonía. IESA. Caracas 1986.

PARAda, N.: Vísperas y comienzos de la revolución de Cipriano Castro. Caracas 1973.

PARDo, Isaac: Esta tierra de gracia. Ed. Dimensiones. 1980.

Parra Pérez, C.: Mariño y las guerras civiles. 3 vols. Ed. Cultura Hispánica. Madrid 1958-1960.

-: Historia de la Primera República de Venezuela. Ed. Guadarrama. Madrid 1959.

Perera, Ambrosio: Historia Orgánica de Venezuela. Caracas 1943.

Pérez, Ana M.: La verdad inédita. Ed. E.v. Armitano. Caracas 1975.

PÉREZ, Joseph: Los movimientos precursores de la Emancipación en Hispanoamérica. Ed. Alhambra. Madrid 1977. 
Pérez VILA, Manuel: Perspectiva del siglo XIX venezolano. Ed. Universidad Santa María. Caracas 1984.

PICON SALAS, M.: Venezuela independiente: evolución política y social, 1810-1960. Caracas 1962.

—: Las ideas de Cipriano Castro. Caracas 1953.

PINO ITURRIETA, Elías: La mentalidad venezolana de la Emancipación (18101812). Instituto de Estudios Hispanoamericanos. Univ. Central de Venezuela. Caracas 1971.

RODRIGUEz, J. S.: Contribución al estudio de la guerra federal en Venezuela. 2 vols. Caracas 1960.

Salcedo Bastardo: Historia fundamental de Venezuela. Univ. Central de Venezuela. Caracas 1982.

Siso, J. M.: Ciento cincuenta años de vida republicana. Ed. Ministerio de Educación. Caracas 1968.

SORIANo, Graciela: Simón Bolívar. Escritos políticos. Alianza Editorial. Madrid 1983.

Uslar Pietri, Arturo (Prólogo); Caldera, Rafael (Prólogo), y otros: Bolivar, Bell y el porvenir. Fundación Eugenio Mendoza. Caracas 1981.

VelAzQuez, R. J., y otros: Venezuela moderna. Medio siglo de historia, 1926-76. Ed. Ariel. Madrid 1979.

Verzna, Paul: Petion y Bolivar. Ed. de la Presidencia de la República. Caracas 1980.

WALTERS, M.: A history of the Church in Venezuela, 1810-1930. Chapel Hill. N.C. 1930.

YANES, Fco. Javier: Compendio de historia de Venezuela. Academia Nacional de la Historia. Caracas 1944. 


\section{CONSTITUCIÓN}

ACAdemIa NaCIONAL DE LA Historia: El pensamiento constitucional hispanoamericano hasta 1830. Compilación de Constituciones. 6 vols. Academia Nacional de la Historia. Caracas 1961.

Acosta, Julio César: El Poder Judicial en la Venezuela Democrática. Repertorio Forense. Tercer Trimestre. 1979.

AL̇ornoz, Orlando: Desarrollo político en Venezuela. Universidad Central de Venezuela. Caracas 1974.

Allen, Henry: Venezuela, una democracia. Ed. Claridad. Buenos Aires 1945.

Alvarado, L.: Historia de la Revolución Federal en Venezuela. 5 vols. Caracas 1953-58.

ANDRADE, I.: Por qué triunfó la Revolución Restauradora. Caracas 1955.

Angulo FreYtes, E.: Situación actual de la acción de Amparo en Venezuela. Univ. Central de Venezuela, Caracas 1979.

ARISMENDI, Alfredo: Contribución a la bibliografía del Derecho Constitucional y su historia. UCU Caracas, 1972.

Baloyra, E., y MARTz, J.: Political Attitudes in Venezuela societal cleavages and public opinion. Univ. of Texas. Austin 1979.

Barnola, Pedro Pablo: (Estudio preliminar) Textos oficiales de la Primer República de Venezuela. Ed. Guadarrama. Madrid 1959.

BarRios FerRer, Gonzalo: “Notas sobre el sistema político venezolano, desarrollo, declive y perspectivas. Mundo Nuevo, n. ${ }^{\circ} 36-37$. Caracas 1987.

—: La imperfecta democracia. Ed. Centauro. Caracas 1981.

BARRIOS, Sonia: «Realidades y mitos de la descentralización gubernamental», en Cuadernos del Cendes. Caracas 1981.

BARTOLETTI, A. M., y otros: Léxico constitucional bolivariano. Edizioni Scientifiche italiana. Napoli 1983. 
BELAÚNDE, Víctor Andrés: Bolivar y el pensamiento político de la revolución hispanoamericana. Talleres Gráficos P. Villanueva. Lima 1977.

Betancourt, Rómulo: «Al comienzo del régimen constitucional», en La Revolución Democrática en Venezuela. Imp. Nacional. Caracas 1968.

—: Defensa del sistema democrático. Imp. Nacional. Caracas 1963.

Blanco MiÑol, Agustín: El 23 de enero: habla la conspiración. Ed. Ateneo. Caracas 1980.

BLANCO, Carlos: «El rol del Estado y del sector privado dentro de una nueva estrategia", en Estado y Reforma, n. ${ }^{\circ}$ 2. Caracas 1987.

Bonilla, F., y Silva Michelena, J. A.: «Cambio político en Venezuela. Exploraciones en análisis y síntesis", en Cuadernos del Cendes. Caracas 1967.

BREWER, C., y AlLAN, R.: Cambio político y reforma del Estado en Venezuela. Ed. Tecnos. Madrid 1975.

-: “Comentarios a la Ley Orgánica de Amparo sobre Derechos y Garantias constitucionales de Venezuela", en Boletín Mexicano de Derecho Comparado, n. ${ }^{\circ}$ 63. U.N.A.M. México 1988.

—: El Estado de Derecho y control judicial. Caracas 1987.

-: El Amparo Constitucional en Venezuela. Instituto de Estudios Jurídicos del Estado. LARA. Barquisimeto, 1987.

-: El Estado incomprendido. Reflexiones sobre el sistema político y su reforma. Vadell Hermanos. Caracas 1985.

-: Instituciones politicas y constitucionales. Ed. Juridica Venezolana y Univ. de Tachira. Caracas 1985.

Bevi Celli; Maro Tulio, y otros: «La Democracia venezolana», en Estado y Reforma, n. ${ }^{\circ}$ 1. Caracas 1986.

Burgraf, W. S.: «The state and institutions of Venezuela from 1936 to 1945 ", en Hispanic American Historical Review. 1989 (v. 69).

BushmELL, D.: The Santander Regime in Gran Colombia. Newark. New York 1954. 
Caldera, R.; Pérez, C. A., y otros: El liderazgo nacional. Comisión Presidencial para la reforma del Estado. Caracas 1986.

CARABACA, Emilio: Diccionario venezolano del político y del parlamentario. Ed. Calvin. Caracas 1983.

Caraillo Batalla, Tomás: Historia critica del concepto de la democracia. Ed. Monte Ávila. Caracas 1983.

CatalA, J. A. (Comp.): Pérez Jiménez y su régimen de terror, 1948-1952. Ed. Centauro. Caracas 1977.

Comision Presidencial para la Reforma del Estado: Propuestas para reformas politicas inmediatas. Caracas 1986.

Congreso de la República: Fuentes para el estudio del 23 de enero de 1958. Caracas 1984.

CORIAT, C.: Inicio y desarrollo de la democracia. Ed. Fundarte. Caracas 1981.

Crespo de Hernandez, Ch.: "Proyecto de Ley de la carrera judicial», en Revista del Consejo de la Judicatura, n. ${ }^{\circ}$ 16. Caracas 1980.

CUENCA, Humberto: “Exposición de motivos y documentos sobre el Proyecto de Ley de la carrera judicial», en Revista del Consejo de la Judicatura, n. $^{\circ}$ 15. Caracas 1979.

Escovar Salón, Ramón: El Amparo en Venezuela. Caracas 1971.

—: Orden político e historia de Venezuela. Italgráfica. Caracas 1966.

EWELL, Judith: The Indirectment of a Dictator. Univ. Press. Texas 1981.

-: Venezuela: A Century of Change. Univ. Press. Stanford, 1984.

Flores, Carlos M.: Gómez, patriarca del crimen. El terror y el trabajo forzado en Venezuela. Ed. Ateneo. Caracas 1980.

Garcia Ponce, G., y Camacho Barrios, E.: Diario de la resistencia y la dictadura, 1948-58. Ed. Centauro. Caracas 1982.

—: El diario secreto de la dictadura. Seleven. Caracas 1980. 
GIL Fortoul: Historia Constitucional de Venezuela. 3 vols. Talleres Cesgraf. Caracas 1967.

GIL YEPES, José A.: The challenge of Venezolan democracy. Transaction Books: Brunswick 1981.

Grases, Pedro: Libros y libertad. Eds. Presidencia de la República. Caracas 1974.

Grases, P., y Pérez VILA, M. (incluye texto de R. Betancourt): Documentos que hicieron historia. Siglo y medio de vida republicana (1810-1961). 2 vols. Eds. Presidencia de la República, Caracas 1962.

Grases, P., y Polanco, T.: Simón Bolivar y la ordenación del Estado. Eds. Colegio Universitario Fco. Miranda. Caracas 1979.

Guerra, G.: Democracia y Estado contemporáneo. Ed. Juve. Caracas 1983.

-: La Constitución de 1961. Balance y perspectiva. Univ. de Zulia. Caracas 1987.

GURUTTS, Mijail: «Espinoso camino de la democracia venezolana», en América Latina, n. ${ }^{\circ}$ 3. Moscú 1988.

HARTLYN, J.: «Democracy in Latin-America. Colombia and Venezuela», en Journal of Latin Americas Indies, v. 21. Octubre, 1989.

Hernandez, Carlos Raúl: «Democracia y mitología revolucionaria. Proceso del poder en Venezuela», en La enseñanza viva. Caracas 1978.

-: «Justificación de la reforma del Estado en Venezuela», en Estado y reforma, n. ${ }^{\circ}$. Caracas 1986.

HeRRERA CAMPINS, Luis: “La transición política», en Tránsito de la dictadura a la democracia en Venezuela. Ed. Ariel. Caracas 1978.

HowARD, Sabin: Rómulo Gallegos y la revolución burguesa en Venezuela. Ed. Monte Ávila. Caracas 1976.

IRAZÁBAL, Carlos: Hacia la democracia. Contribución al estudio de la historia económica, política y social en Venezuela. Ateneo. Caracas 1979. 
Josko, Eva: «El Congreso y la política exterior de Venezuela», en Politeia, n. ${ }^{\circ}$ 97. 1978.

-: «La Política Exterior: continuidad y cambio, contradicción y coherencia", en El caso Venezuela. Eds. IESA. Caracas 1986.

KoRNBLITH, M., y MAINGON, T.: «Contenido y formas de acción estatal en el periodo 1936-80", en Cuadernos del Cendes, n. ${ }^{\circ} 4$. Caracas 1987.

Labarca Prieto, Domingo: Bolivar y la organización del poder público. Ed. Maracaibo. Maracaibo 1981.

LEONI, Raúl: Documentos Presidenciales. Oficina Central de Información. Caracas 1969.

Levine, D.: Conflict and political change in Venezuela. Princeton. Univ. Press. Princeton 1973.

-: "Venezuela since 1958: the consolidation of Democratic Politics", en The Breakdown of Democratic Regimen: Latin America. Ed. por J. Linz, y A. Stephan, Baltimore the John Hopkins. Univ. Press, 1978.

LiNARES BeWZo, Gustavo: «El proceso de Amparo en Venezuela», en Revista de la Fundación Procuraduría General de la República, n. ${ }^{\circ}$ 2. 1987.

López Maya, M., y Gómez Camaño, L.: "Crisis y concertación en Venezuela. Dos coyunturas históricas" en Concertación político social y democratización. CLACSO. Buenos Aires 1987.

López Orimuela, Alcides: El pacto y las democracias sólidas. Eds. Greco. Caracas 1986.

LOVERA ReYES, E.: «Autonomismo y realismo en la Provincia de Coro durante la independencia", en Anuario de Estudios Bolivarianos. Año $1, \mathrm{n}^{\circ} 1$. Univ. Simón Bolivar. 1990.

LUCIANI, Jorge: La dictadura perpetua de Gómez y sus adversarios. Lersive y Rosboro. New York 1930.

LYNCH, John: «Bolivar and the Caudilles", en Hispanic American Historial Review, 63. 1983.

MARTZ, J., y MYERS, D.: Venezuela, the democratic experience. Praeger. New York 1977. 
Medina Anganita, Isaias: Cuatro años de democracia. Pensamiento. Caracas 1963.

MiJARES, Silvia: Organizaciones politicas de 1936: su importancia en la solidificación política del venezolano. Ed. Academia Nacional de Historia. Caracas 1980.

MINISTERIO RELACIONES INTERIORES DE VENEZUELA: El Gobierno revolucionario de Venezuela ante su pueblo. Imprenta Nacional. Caracas 1949.

Monsisalve, José D.: Actas de la Diputación Permanente del Congreso de Angostura. Imprenta Nacional. Bogotá 1927.

MORENo, Óscar: «El Estado y la cuestión regional en Venezuela», en Cuadernos del Cendes, n. ${ }^{\circ}$ 4. Caracas 1984.

NJaIM, Humberto: El sistema político venezolano. Instituto de Estudios Políticos. Facultad de Derecho. Univ. Central de Venezuela. Caracas 1975.

OlavarRia, Jorge: Dios y Federación. El fetichismo federal en el pasado, presente y futuro de Venezuela. Fundación para una Nueva República. Caracas 1988.

Oropeza, Ambrosio: La nueva Constitución venezolana. Imprenta Nacional. Caracas 1969.

PEÑA, Alfredo: Democracia y reforma del Estado. Ed. Jurídica Venezolana. Caracas 1978.

-: Corrupción y Golpe de Estado. Ed. Ateneo. Caracas 1980.

Pérez, Carlos Andrés: El país frente a sus realidades. Ed. Centauro. Caracas 1980.

-: «La reforma del Estado», en Nueva Sociedad. n. ${ }^{\circ}$ 79. Caracas 1985.

PORTALES, Carlos: «Democracia y politica de integración", en El caso Venezuela. FLACSO. Santiago de Chile 1981.

Presidencia de LA República: Documentos que hicieron historia. Siglo y medio de vida de la República, 1810-1960. 2 vols. Caracas 1962.

Quevedo, N.: El Gobierno Provisorio. P.V.R.O. C. Bolivar, 1958. 
Ramirez Farias, Carlos: La democracia petrolera. De Rómulo Betancourt a Carlos Andrés Pérez. CIP. 1978.

Ramos, A.; Combellas, R.; Rincón, E., y otros: Venezuela un sistema político en crisis. KAPPA Editores. Mérida 1987.

Rodriguez, Luis C.: «Gómez y el anticomunismo», en Tierra Firme, n. ${ }^{\circ} 12$. Caracas 1985.

Rodriguez Cruz, Francis: «Índice cronológico de leyes, decretos y resoluciones publicadas en la Gaceta Oficial de la República en el año 80", en Anuario, n. ${ }^{\circ}$ 12. Universidad de los Andes. Mérida 1982.

-: «La Constitución, el Poder Judicial, la función jurisdiccional y la judicatura», en Anuario, n. ${ }^{\circ}$ 12. Universidad de los Andes. Mérida 1982.

Romero, Aníbal: La miseria del populismo. Mitos y realidades de la democracia en Venezuela. Eds. Centauro. Caracas 1986.

ROMERo, Carlos: “Las empresas y el sistema político venezolano. Las reglas del juego», en Politeia, n. ${ }^{\circ} 11$. Caracas 1982.

SALAZAR, Diego: Veinte años de lucha revolucionaria. Los últimos días de Pérez Jiménez. Ruptura. Caracas 1978.

SCHATTSCHNEIDER, E. E.: El pueblo semi-soberano. UTEHA. México 1967.

Silva Michelena, J. M.: Crisis de la Democracia. Univ. Central de Venezuela. Caracas 1970.

-: El sistema politico venezolano. Universidad Central de Venezuela. Caracas 1975.

-: «Evaluación general de la política gubernamental durante el período 1979-83", en Cuadernos del Cendes. Vol. 1, n. ${ }^{\circ} 1.1983$.

SONNTAG, Heinz: «Estado y desarrollo sociopolítico en Venezuela, en Cuadernos del Cendes, n. ${ }^{\circ}$. Caracas 1984.

—: «La burocracia a la defensiva», en Estado y Reforma. Noviembre, 1987. Caracas.

SosA, Arturo: Ensayos sobre el pensamiento político positivista venezolano. Ed. Centauro. Caracas 1985. 
Stamboli, Andrés: Crisis política. Venezuela, 1945-1958. Ed. Ateneo. Caracas 1980.

Stempel Paris, Antonio: Venezuela, una democracia enferma. Apuntes para el estudio del desarrollo político venezolano. Ed. Ateneo. Caracas 1981.

TORRES, Arístides: «Pautas de la evolución del sistema político democrático en Argentina y Venezuela", en Mundo Nuevo, n. ${ }^{\circ}$ 36-37. Caracas 1987.

Tovar Tamayo, Orlando: «El Régimen Político venezolano», en Revista de Derecho Político, n. ${ }^{\circ} 32$. Madrid 1991.

Universidad Central de Venezuela: «Estado y proceso sociopolíticos en Venezuela», en Cuadernos del Cendes, n. ${ }^{\circ}$ 4. Caracas 1984.

URBANEJA, Diego Bautista: El sistema político o cómo funciona la máquina de procesar decisiones. Ed. IESA. Caracas 1986.

-: «Introducción histórica al sistema político venezolano», en Politeia, n. ${ }^{\circ} 7.1978$.

-: «Perspectivas ideológicas de la democracia», en Venezuela, 1979. Examen y Futuro. Ateneo. Caracas 1980.

Valenilla Lanz, Laureano: Cesarismo Democrático. Tip. Garrido. Caracas 1961.

Velasquez, T.; Sucre Figarella, J. F., y Bruni Celli, B.: Bentancourt en la historia de Venezuela del siglo XX. Ed. Centauro. Caracas 1980.

VelAzQUEZ, Ramón: Aspectos de la evolución política en Venezuela en el último medio siglo. Fundación Empresa Mendoza. Caracas 1976.

WEIZZ, Nikolaus: 25 jahre wanhlen mal demokratic in Venezuela. Heidelberg Britte Welt Studien. Heidelberg 1984.

\section{PROCESOS ELECTORALES}

Álvarez, Federico: La izquierda venezolana y las elecciones de 1973. Sint. Dos Mil. Caracas 1974. 
Bravo, Argelina: Crisis, elecciones y violencia. Ruptura. Caracas 1978.

Buminov PARRA, Boris: Introducción a la sociología electoral venezolana. Ed. Arte. Caracas 1968.

-: "Legislación electoral venezolana", en Legislación electoral comparada. Eds. CAPEL. San José de Costa Rica 1986.

BUSHELL, D.: «La evolución del derecho de sufragio en Venezuela», en Boletín Histórico, 39. Caracas 1972.

Carvallo, G., y Rios de Hernandez, J.: «Dominación burguesa y democracia representativa en Venezuela: Apuntes para la evaluación de su funcionamiento", en Revista Mexicana de Sociología. Vol. XLIII, n. ${ }^{\circ} 2$. 1981.

Combellas, R.; ReY, Juan Carlos, y otros: Reformas electorales y partidos políticos. Consejo Supremo Electoral. Caracas 1986.

CHANG, M. Roberto: Sistemas y cifras de las elecciones venezolanas desde 1958. C.S.E. Caracas 1980.

Garcia Laguardia, J. M.; NJaim, H., y otros: Sistemas electorales. Acceso al sistema político y sistema de partidos. Consejo Supremo Electoral. Caracas 1987.

Garcla Ponce, Guillermo: El país, la izquierda y las elecciones de 1978. Talleres TIP. M. A. García. Caracas 1977.

Madrazo Cuellan, J. L.: «Estudio comparativo de la legislación electoral centroamericana y de Colombia, México, Panamá y Venezuela», en Legislación Electoral Comparada. Eds. CAPEL. San José de Costa Rica 1986.

MARQUez Pompeyo: La actuación de los partidos, la farsa electoral y el bloque único. Liberación Nacional. Caracas 1950.

MARTELLO, Mercedes: «El sistema electoral venezolano. Resultado de la aplicación del sistema de Representación Proporcional en la composición de las Cámaras del Congreso", en Propaganda, política, partidos y sistema electoral. Publicación del Consejo Supremo Electoral. Caracas 1987. 
Molina, José Enrique: «Democracia representativa y participación política en Venezuela», en Cuadernos Capel, $n .^{\circ} 10$. San José de Costa Rica 1986.

OropeZA, L. J.: El pluralismo tutelar. Ed. Centauro. Caracas 1982.

Petkoff, Teodoro: El 23 de enero de 1958 y el proceso de consolidación de la democracia representativa en Venezuela. GABIZU - Todman. Caracas 1978.

Rodriguez Iturbe, José: Polarización y bipartidismo en las elecciones de 1973. Arte. Caracas 1974.

Salcedo Bastardo, J. L. (Comp.): Bolívar y Sucre. 150 años después. Banco Nacional de Ahorro y Préstamos. Caracas 1980.

Silva Michelena, José A.: El proceso electoral de 1978: su perspectiva histórico estructural. Ed. Ateneo. Caracas 1979.

TORRES, Aristides: Partisanhip and fleating electoral behaviva in Venezuela. Massachussetts Institute of Technology. Cambridge 1983.

TOVAR TAMAYo, Orlando: "Las instituciones electorales en Venezuela», en Sistemas electorales y representación politica en Latinoamérica. Ed. Friedrich Ebert. ICl. Madrid 1986.

Tovar Tamayo, O.; Torres, A.; Keller, A., y otros: Innovación democrática, mitos políticos y organización electoral. Consejo Supremo Electoral. Caracas 1988.

Veloz, M. Armando: Manual electoral. Ed. Profarte. Caracas 1978.

VILLABONa BLANCO, M. P.: “Política y elecciones en Venezuela», en Revista de Estudios Políticos, n. ${ }^{\circ}$ 53. Madrid 1986.

\section{FUERZAS POLITICAS Y SOCIALES}

\subsection{Partidos politicos. Obras generales}

ACEVEdo de SucRe, M. L., y Nones, C.: La generación venezolana de 1928. Estudio de una élite política. Caracas 1967. 
CarTa Ramirez, Gehard: Los partidos políticos 'en Venezuela contemporánea. Imp. Congreso de la República. Caracas 1981.

-: Política y partidos modernos en Venezuela. Especial referencia al socialismo marxista, la socialdemocracia y la democracia cristiana. Ed. Centauro. Caracas 1983.

CODETTA, Carolina: “Notas sobre la dimensión izquierda-derecha en Venezuela», en Mundo Nuevo, n. ${ }^{\circ} 31$. Caracas 1986.

Congreso de la República: Pensamiento Político Venezolano del siglo XIX. Caracas 1983.

DeBay, Regis: Venezuela. Las pruebas de fuego. Ed. Siglo XXI. México 1975.

Grases, P., y Perez VILA, M. (eds.): Pensamiento político venezolano del siglo XIX. 15 vols. Caracas 1960-62.

IZARD, Miguel: «De liberadores a gestores. Algunas notas sobre el conservadurismo venezolano», en Tierra Firme, n. ${ }^{\circ}$ 9. Caracas 1985.

KEY, Fernando: Prisiones de Venezuela a la muerte de Juan Vicente Gómez. Ed. Centauro. Caracas 1974.

Magallanes, M. V.: Los partidos politicos en la revolución histórica venezolana. Ed. Mediterráneo. Caracas 1973.

MART, John D.: «Los peligros de la petrificación. El sistema de partidos venezolanos y la década de los ochenta», en La obra Iberoamérica en los años 80. Perspectivas de cambio social y político. ICl. Madrid 1982.

NJAIM, Humberto: «El sistema venezolano de partidos y campos de influencia», en Politeia, n. ${ }^{\circ} 7.1978$.

NJAIM, H.; Brito, J.; ReY, J. C., y otros: El financiamiento de los partidos políticos y la democracia en Venezuela. Ed. Ateneo. Caracas 1981.

Nuaim, H.; Área, L.; Guevara, P., y otros: Propaganda política, partidos y sistemas electoral. Consejo Supremo Electoral. Caracas 1987.

Pérez Perazo, J. l.: Los partidos políticos latinoamericanos como grupos de presión, principales grupos de presión latinoamericanos. Caracas 1967. 
RANGEL, Domingo Alberto: Los andinos en el poder. Balance de una hegemonía, 1899-1945. Caracas 1965.

REY, Juan Carlos: El financiamiento de los partidos políticos y la democracia en Venezuela. Ateneo Jurídico. Caracas 1981.

-: «El futuro de la democracia en Venezuela», en Venezuela hacia el 2000. Ed. Nueva Sociedad. Caracas 1987.

—: «El sistema de partidos venezolanos», en Politeia, n. ${ }^{\circ}$ 1. Caracas 1972.

—: “ldeología y cultura política: el caso del populismo latinoamericano", en Politeia, n. ${ }^{\circ}$ 5. Caracas 1976.

—: Problemas sociopoliticos de América Latina. Ed. Ateneo. Caracas 1980.

RoBinson, David J.: “La Libertad. La frágil fraternidad y la desigualdad en los primeros tiempos de Hispanoamérica republicana. Una evaluación del impacto de los ideales revolucionarios franceses", en Anuario de Estudios Bolivarianos, n. ${ }^{\circ}$ 1. Universidad Simón Bolivar. 1990.

Rodríguez Gallad, Irene: Venezuela ante el ascenso y caída de la restauración liberal. Ed. Ateneo. Caracas 1980.

Rondón Maraver, R. A.: Guzmán Blanco, el autócrata civilizador. Tip. Garrido. Caracas 1944.

Romero, José Luis: Pensamiento político de la Emancipación, 1790-1825. Biblioteca Ayacucho. Caracas 1977.

Romero, J. L., y Moreno, L. A.: Pensamiento conservador (1815-1898). Biblioteca Ayacucho. Caracas 1978.

Silva, M.: El financiamiento de los partidos políticos y la democracia en Venezuela. Ed. Ateneo. Caracas 1981.

SuARez FigueroA, Naudy: Programas politicos venezolanos de la primera mitad del siglo XX. 2 vols. Universidad Católica Andrés Bello. Caracas 1977.

Suno, Pedro: Sobre aparatos y desviaciones. Nueva Izquierda. Caracas 1969. 
VALLENILLA LANZ, L.: Disgregación e integración. Ed. Fondo Editorial. Cola de Fuenmayor. Caracas 1984.

VelAzQUeZ, Ramón S.: La caida del liberalismo amarillo. Tiempo y drama de Antonio Paredes. Ed. Contraloria General de la República. Caracas 1972.

-: El.pensamiento político del siglo XX. Documentos para su estudio. Caracas 1983.

\subsection{Acción Democrática}

Acción Democrática: Ante la conciencia revolucionaria de América. México 1949.

—: Historia, organización y capacitación. CEDAL. San José 1976.

—: Libro Negro, 1952. Catalá Editor. Caracas 1974.

-: Doctrina y programas. Pub. Sec. Nacional de Propaganda. Caracas 1962.

—: Tesis politica. Departamento Nacional de Prensa y Propaganda. Caracas.

—: Un reto a la dictadura. Ed. Cordillera. Caracas 1959.

ACRATE, Jorge: "Socialismo europeo y sudamericano", en Nueva Sociedad, n. ${ }^{\circ} 72$. Caracas 1984.

AleXANDER, R.: Rómulo Betancourt and the transformation of Venezuela. Transaction Books. New Bruncwick. New York 1982.

Betancourt, Rómulo: El 18 de octubre de 1945. Génesis y realizaciones de una revolución democrática. Seix Barral. Caracas 1979.

-: "VI Mensaje Presidencial», en La Revolución Democrática de Venezuela. Tomo IV. Imprenta Nacional. Caracas 1968.

—: Pensamiento y acción. Silva. México 1951. 
BETANCOURT, Rómulo: Trayectoria democrática de una revolución. Imprenta Nacional. Caracas 1948.

—: Rómulo Betancourt: memoria del último destierro. Ed. Centauro. Caracas 1982.

Bruno Celli, Marco Tulio: Acción Democrática y los primeros programas politicos del plan de Barranquilla o la candidatura simbólica de Rómulo Gallegos, 1941. Ed. Centauro. Caracas 1980.

Caballero, Manuel: Rómulo Betancourt. Ed. Centauro. Caracas 1977.

-: «Reflexiones sobre la historia de la izquierda», en Nueva Sociedad, n. ${ }^{\circ} 61$. Caracas 1982.

Carmona, Luis A.: Aspectos históricos o ideológicos del Partido Acción Democrática. Doctrina. Caracas 1978.

Carpio Castillo, Rubén: Acción Democrática, 1941-1971. Bosquejo histórico de un partido. Eds. República. Caracas 1971.

Carrera Damas, Germán: Para la historia de los origenes del socialismo en Venezuela. Cultura Universitaria. Caracas 1960.

DabAGUIAN, Emil: Venezuela: evolución ideológica y política del partido Acción Democrática. Ac. Ciencia URSS. Instituto América Latina. Moscú 1978.

ELLNER, S.: "Organized Labors political influence and partyties in Venezuela. Accion Democratica and its labor leadership", en Journal of interamerican studies and world affaires. v. 31, n. ${ }^{\circ} 4.1989$.

Fracción Parlamentaria de Acción Democrática: La subversión extremista en Venezuela. Caracas 1964.

GARCIA PONDE, Antonio: ¿Adecos, tucanes o marxistas? Una historia de la izquierda, 1959-1984. Ed. Domingo Fuentes. Caracas 1985.

González Herrera, Luis: Rómulo en Berna. Un documento para la historia de Acción Democrática. Ed. Centauro. Caracas 1971.

LAIRET, Germán: « ¿Es posible el socialismo democrático en América Latina?». Nueva Sociedad, n. ${ }^{\circ} 56$ y 57. Caracas 1981. 
LARDEN, Luis: "La doctrina de Acción Democrática», en Cuadernos Americanos, n. ${ }^{\circ}$ 4. México 1950.

LusINCHI, J.: Contra la crisis, sacrificios fructiferos y acción creativa. Ministerio de la Secretaría de la Presidencia. Caracas 1984.

-: El pais es uno solo. Discursos del Presidente de la República. Ministerio de la Secretaría de la Presidencia. Caracas 1984.

MARTS, John D.: Accion Democratica: evolution of a modern political party in Venezue/a. Princeton. Univ. Press. 1966.

MONGE, Luis Alberto: Evolución de la idea socialdemócrata en América Latina y Costa Rica. Cepal. San José 1976.

Peña, Alfredo: Conversaciones con Carlos Andrés Pérez. Ed. Ateneo. 1979.

Rivas Rivas, José: Las tres divisiones de Acción Democrática. Pensamiento vivo. Caracas 1958.

SerXUER, Stanley: Accion Democratica of Venezuela: its origin and development. Gainville. Univ. Floria 1959.

SosA, Arturo: Del garibalismo estudiantil a la izquierda criolla. Los orígenes marxistas del proyecto de Acción Democrática (1928-1935). Ed. Centauro. Caracas 1981.

TAfre Murzi, Alfredo: Los adecos en el poder. Seleven. Caracas 1983.

\subsection{C.O.P.E.I. (Social Cristianos)}

CALDERA, Rafael: "América Latina: la democracia y Europa», en Politica Internacional, n. ${ }^{\circ}$ 5. Caracas 1987.

-: "En la toma de posesión de la primera magistratura", en Metas de Venezuela. Tomo I. Oficina Central de Información. Caracas 1970.

-: La actitud del socialcristianismo en defensa de la democracia. COPEI Americana. Caracas 1960.

-: La solidaridad pluralista en América Latina. Caracas 1973. 
Caldera, R.; Velázouez, R.; Petkoff, T., y otros: Sobre la Democracia. Ed. Ateneo. Caracas 1979.

CoMBELLA, Ricardo: COPEI, ideología y liderazgo. Ed. Ariete. Caracas 1985.

Gimenez Landinez, Víctor: Los copeyanos. Ed. Centauro. Caracas, 1982.

GuRUITS, Mijail: «El partido socialcristiano COPEI y la transformación del sistema político venezolano», en América Latina, n. 5 . Nuevo México 1982.

Herrera Campins, L.: «Discurso pronunciado en el acto de toma de posesión», en Política Exterior de Venezuela. T. I. Ministerio de Relaciones Exteriores. Caracas 1980.

—: Frente a 1958. Documentos CIDAL. Caracas 1957.

-: Voz y camino: 24 editoriales de oposición. Ed. Ruta, Voz y Camino. Caracas 1986.

—: Violencia y politica. Ed. Monte Ávila. Caracas 1972.

MAGGı CooK, Santiago: «Formación democrática en América Latina; la contribución venezolana de Acción Democrática y COPEI», en Nueva Sociedad, n. ${ }^{\circ}$ 34. Caracas 1978.

MoLT, Peter: La programática política de los partidos demócratas cristianos de la América Latina. CIDAL. Caracas 1967.

MYERS, David J.: Democratic campigning in Venezuela: Caldera's Victory. Fund. La Salle de Ciencias Sociales. Caracas 1973.

PAdRón, Paciano: COPEI. Documentos fundamentales. Ed. Centauro. Caracas 1981.

Partido Social Cristiano COPEl: COPEl. 25 años. Caracas 1971.

-: Los diecinueve años de COPEI. Caracas 1965.

-: Programa y Estatutos. Caracas 1948.

RIVERA OVIEDo, J. E.: Los socialcristianos de Venezuela: historia e ideología. Ed. Centauro. Caracas 1977. 
StIELER, Hans Günter: «Zur Ideologie und Politic der Christhian sozialen Partei COPEI in Venezuela», en Lateinamerika, n. ${ }^{\circ}$ 1. Rostock 1987.

\subsection{Partido Comunista de Venezuela}

AleXAnder, Robert: El Partido Comunista de Venezuela. Diana. México 1971.

—: La revolución democrática de Venezuela. Albón Interp. Medellín 1967.

BoERSNer, Demetrio: Análisis del Partido Comunista en Latinoamérica y Venezuela. Univ. Popular Alberto Carnevali. Caracas 1961.

Caballero, Manuel: La Internacional Comunista de América Latina. La Sección Venezolana. Ed. Pasado y presente. México 1978.

Castro, P., y Pases, C.: El Partido Comunista de Venezuela: browderismo y colaboración de clases. Univ. Central de Venezuela. Caracas 1979.

CoRTA, José: Frente al Comunismo ateo. Hechos y dichos. Caracas 1962.

oE la Plaza, S., y Duclós, J.: Antecedentes del revisionismo en Venezuela. Fondo Editorial Salvador de la Plaza. Caracas 1973.

DIaz Rangel, Eleazar: «Cómo se dividió el P.C.V.». Ed. Domingo Fuente. Caracas 1971.

Farias, Jesús: Los Comunistas en el Parlamento, 1959-1963. Caracas.

FuenMAYOR, Juan Bautista: Aportes para la historia del P.C.V.». Maracaibo 1971.

-: El papel de la clase obrera y de los comunistas en la etapa actual. Tamanaco. Caracas 1945.

Gallegos Manlerca, E.: «Esquema cronológico de la historia del Partido Comunista de Venezuela", en Ideologia, n. ${ }^{\circ} 3$ y $4,\left(2 .^{a}\right.$ etapa). Caracas 1981 y 1982.

GaRCIA PONCE, Guillermo: Cinco temas de organización sobre la estrategia revolucionaria en Venezuela. Cantaclaro. Caracas 1969. 
Garcia Ponce, Guillermo: El túnel de San Carlos. La Muralla. Caracas 1968.

-: Introducción a la política venezolana. Com. Nal. de Educación del P.C.V. Caracas 1961.

—: Política, táctica y estrategia. La Muralla. Caracas 1967.

-: Politica y clase media. La Muralla. Caracas 1966.

-: Qué es el Partido Comunista de Venezuela. Suplemento de Cuadernos Comunistas. Caracas 1960.

—: Teoría política y realidad nacional. La Muralla. Caracas 1967.

GÓmEz, Alejandro: «Las distintas tendencias en el Comunismo venezolano, 1943-1946", en Clase obrera, partidos y sindicatos en Venezuela. Ed. Centauro. 1982.

MACHADO, Gustavo: Los comunistas acusan. El betancurismo en el banquiIlo. Tribuna Parlamentaria. Caracas 1969.

-: Siempre junto al pueblo y leales a la unidad. Gráfica Americana. Caracas 1958.

MÁrqueZ, Pompeyo: “Una polémica necesaria: Fidel Castro. P.C.V.», en Documentos Políticos. 1968.

Partido Comunista de Venezuela: «Creación del Partido Comunista de Venezuela", en Documentos que hicieron historia. Presidencia de la República. Caracas 1962.

—: El leninismo y la liberación de la mujer. Ed. Cantaclaro. Caracas 1970.

-: Estatutos. Caracas 1980.

—: Frente Patriótico para derrotar a la agresión imperialista. Gráfica Orinoco. Caracas 1975.

-: Primer Congreso de Unidad de los Comunistas. Comisión Nacional de Educación y Propaganda. Caracas 1946.

-: Problemas candentes del movimiento revolucionario venezolano. México 1967. 
Partido Comunista de Venezuela: Programa del Partido Comunista de Venezuela. Río Orinoco. Caracas 1974-89.

ReY SANCHEZ, Fernando: Fundación del Partido Comunista de Venezuela. Fondo Editorial C. Apuntes: Caracas 1984.

Robledo LIMÓN, Ricardo: «El Partido Comunista de Venezuela, sus técnicas políticas de 1964 a 1969", en Foro Internacional. Vol. XI, n. ${ }^{\circ} 4$. México 1971.

\subsection{M.A.S.}

Cortez, José Rafael: El M.A.S., desbandada hacia la derecha. Ed. Centauro. Caracas 1971.

ELLNER, Seteve: "The M.A.S. party in Venezuela», en Latin America Perspectives, n. ${ }^{\circ}$ 2. R.V. 1986.

MARQUEZ, Pompeyo: El M.A.S., fuerza clave en la política venezolana. Punto Socialista. Caracas, octubre 1977.

-: «EI M.A.S. hace historia», en Punto Socialista, n. ${ }^{\circ}$ 8. Caracas, enero, 1984.

—: Hacia una nueva mayoría. Equipo Editor. Caracas 1979.

-: Hacia una victoria del socialismo. Caracas 1973.

-: Santos Yorme o Pompeyo Márquez, combatiente sin tregua. Ed. Centauro. Caracas 1979.

—: Socialismo en tiempo presente. Ed. Centauro. Caracas 1973.

Peña, Alfredo: Conversaciones con José Vicente Rangel. Ed. Ateneo. Caracas 1978.

Petkoff, Teodoro: Corrupción total. Ed. Fuentes. Caracas 1979.

—: Democracia para el socialismo. Socoraime. Caracas 1981. 
Petkoff, Teodoro: «El movimiento del socialismo (M.A.S.) de Venezuela», en Revista Mexicana de Sociologia, n. ${ }^{\circ}$ 3. UNAM. México 1981.

-: El socialismo, una necesidad impostergable. Ed. Fuentes. Caracas 1976.

-: «Hacia un nuevo socialismo», en Nueva Sociedad, n. ${ }^{\circ} 56-57$. Caracas 1981.

-: Origen e historia del M.A.S. Cumaná 1982.

-: Proceso a la izquierda. Ed. Planeta. Caracas 1976.

-: ¿Socialismo para Venezuela? Ed. Fuentes. Caracas 1972.

Rangel, D.: Venezuela y el socialismo M.A.S. Caracas 1978.

\subsection{Otras fuerzas políticas}

AcuÑa, Guido: Cuando mataron a Ruiz Pineda. Ed. Arévalo González. Caracas 1947.

ANNEZ, Jacinto: Gustavo Machado de oligarca a comunista, 1914-1974. Ed. Centauro. Caracas 1975.

BUTLER, R. W.: The origins of the Liberal Party in Venezuela, 1830-1848. Univ. of Texas. 1972.

CARRERA, G.: Historiografia marxista venezolana y otros temas. Universidad Central de Venezuela. Caracas 1967.

CatalA, José Agustín: Venezuela bajo el signo del terror, 1948-52. Caracas 1979.

CoRtez, José Rafael: En defensa del socialismo. Gráfica Orinoco. Caracas 1980.

Delgado Chalbaud, Miguel: Anotaciones para el programa del Partido Nacional Laborista de Venezuela. Caracas 1936. 
Diaz Seluas, Pedro: Luis Beltrán Prieto, líder de un pueblo. Costa Amic. México 1968.

—: El Partido Demócrata Venezolano y su proceso. Ed. Elite. Caracas 1938.

Franceiche, Alberto: Ochenta reflexiones, tesis e hipótesis del M.I.R. y para el M.I.R. Tendencia Proletaria. Carabobo 1979.

Gallego, Rómulo: Una posición ante la vida, 1948-54. Ed. Centauro. Caracas 1977.

GonZÁLEZ, Juan Vicente: Juan Vicente González. La doctrina conservadora. Presidencia de la República. Caracas 1960.

Heydora, Pastor: La izquierda, una autocrítica perpetua. Univ. Central de Venezuela. Caracas 1981.

Instituto AUtÓnOMo, Biblioteca Nacional: Los liberales amarillos en la caricatura venezolana. Caracas 1982.

LANZ, Roberto: Por una teoría del poder y del partido. Ed. Ateneo. Caracas 1979.

LARRAZABAL, Radalmes: Sobre la revolución: teoria de intelectuales y práctica revolucionaria. Ed. Cantaclaro. Caracas 1976.

Liga Socialista de Venezuela: «¿Qué partido construir?, en Teoría Política, n. ${ }^{\circ}$ 11. México 1983.

MARTIN, Américo: El socialismo no es una religión. Ed. Ateneo. Caracas 1979.

MARTínez, Pedro: La unidad de la izquierda en Venezuela. Instituto de Estudios Políticos. Caracas 1980.

MoleIRo, Moisés: El M.I.R. de Venezuela. La Habana 1968.

-: La izquierda superada. Ed. Ateneo. Caracas 1983.

-: La izquierda y su proceso. Ed. Centauro. Caracas 1977.

Muñoz, Fredy: Revolución sin dogma. Universidad Autónoma de Sinaloa. Culciacán 1971. 
NúnEZ, Tenorio: La izquierda y la lucha por el poder en Venezuela. Ed. Ateneo. Caracas 1979.

—: ¿Qué es la nueva alternativa? Ed. U. 83. Caracas 1983.

-: Sobre vanguardias y revolución socialistas. Cabima. Caracas 1972.

-: Venezuela y la revolución socialista. Universidad Central de Venezuela. Caracas 1976.

-: Venezuela neocolonial: justicia social para ser realmente libres. Univ. Central de Venezuela. Caracas 1969.

OJEDA, Fabricia: Hacia el poder revolucionario. Guairas. La Habana 1967.

-: La guerra del pueblo. Ed. Fuentes. Caracas 1970.

Paredes, A.: ¿Cómo llegó Cipriano Castro al poder? Caracas 1954.

Partido Democrático Venezolano: El Partido Democrático Venezolano y su proceso. Ed. Elite. Caracas 1938.

PARtido Revolucionario del Proletariado: La revolución y la estrategia revolucionaria. Caracas 1947.

-: La unidad de acción de los trabajadores de Venezuela. Ávila Gráfica. Caracas 1952.

Peña, Alfredo: Conversaciones con Luis Beltrán (M.E.P.). Ed. Ateneo, 1978.

Rangel, D.: Expediente Negro. Ed. Fuentes. Caracas 1969.

—: Gómez el amo del poder. Vadell. Valencia 1975.

-: La revolución de las fantasias. OFIDI. Caracas 1966.

-: Una teoría para la revolución democrática. Ed. Arte. Caracas 1958.

\subsection{Sindicatos y organizaciones obreras}

Castro, Pedro: Movimiento obrero en años de represión. Ed. Centauro. Caracas 1982. 
Delgado Bello, Luis: “El movimiento cooperativo y los procesos de democratización”, en Socialismo y participación, n. ${ }^{\circ}$ 36. Lima 1986.

Dominguez, Raúl: "Un siglo de luchas políticas campesinas en Venezuela», en Historia Politica de los campesinos latinoamericanos. Instituto de Investigaciones Sociales. UNAM. Siglo XXI. México 1985.

ELLNER, Seteve: Los partidos políticos y su disputa por el control del movimiento sindical en Venezuela, 1936-1948. Universidad Católica Andrés Bello. 1980.

Dos Santos, M.; Rial, J.; Riz, L., y otros: Concertación política, social y democratización. CLACSO. Argentina 1987.

Godıo, Julio: Cincuenta años de la C.T.V. (1936-86). Historia, doctrina y acción. ILDIS. Caracas 1986.

-: El movimiento obrero venezolano, 1965-80. Ed. Ateneo. Caracas 1982.

GonzAlez Navarro, José: Democracia y sindicalismo. Vanguardia Obrera. Caracas 1964.

LOPEZ MAYA, M., y NERÓN, N.: El Estado venezolano y el movimiento sindical. Ed. Centro Es. Investigación Científica. Caracas 1981.

Magallanes, Manuel: Augusto Malavé Villalba, ejemplo y símbolo del sindicalismo venezolano. Caracas 1978.

OlARTE, César: "Venezuela, desafíos y propuestas de la C.T.V.», en Nueva Sociedad, 83. Caracas 1986.

SalamanCA, Luis: «El papel de la C.T.V. en el sistema político venezolano. La hipótesis corporativista», en Politeia, n. ${ }^{\circ} 11$. Caracas 1982.

TORRES, Eloy: Ideología y sindicalismo. Caracas 1970.

QUINTERO, Rodolfo: La clase obrera y la revolución. Valerio Cruz. Caracas 1970.

WENZ, Nïkolaus: El Estado y el movimiento sindical (1958-1980). Ed. Cendes. Caracas 1981. 
5.8. Fuerzas Armadas (incluye Movimientos Guerrilleros)

AgüERo, P., y IWONKA, Felipe: «Militares y Democracia en Venezuela», en Sintesis, n. ${ }^{\circ}$. Madrid 1988.

Aguilar, P.; Rangel, J. V., y otros: «Foro, poder civil y poder militar en Venezuela», en Punto Socialista, n. ${ }^{\circ}$ 45-46. Caracas 1987.

Avendaño Lugo, Jr.: El militarismo en Venezuela: la dictadura de Pérez Jiménez. Ed. Centauro. Caracas 1982.

BeltRÁN, J. Rafael: El papel público y social de las Fuerzas Armadas en América Latina. Ed. Monte Ávila. Caracas 1970.

BIGLER, G.: «Professional soldiers and Restrained Politics in Venezuela», en New Military Politics in Latin America. Ed. Robert Wessan Praeger. New York 1982.

-: The Armed Forces and patterns of civil-military relations en Venezuela: the Democratic Experience. Ed. John D. Martz y David J. Myers Praeger. New York 1977.

-: «Venezuela», en The Latin America Military Institution. Ed. Robert Wesson Praeger. New York 1986.

Blanco Muñoz, Agustín: El 23 de enero. Habla la conspiración. Univ. Central de Venezuela. Caracas 1980.

-: La lucha armada, hablan cinco jefes. Universidad Central de Venezuela. Caracas 1980.

-: La lucha armada, hablan seis comandantes. Universidad Central de Venezuela. Caracas 1981.

-: La lucha armada: la izquierda revolucionaria insurgente. Universidad Central de Venezuela. Caracas 1981.

Burggraff, Winfield: The Venezuela Armed Forces in politics, 1935-1959. Univ. Missoury Press: 1972.

Calello, Hugo: Poder Militar y Estado Nacional en América Latina. Ed. Rocinante. Caracas 1977. 
Carillo Batalla, T.: Las Fuerzas Armadas de Venezuela en el siglo XIX. 12 vols. Caracas 1963.

CORREA, Luis: F.A.L.N.: Brigada UNO. Fuentes. Caracas 1973.

DAGER, J.: Testigo de excepción en las trincheras de la resistencia, 19481955. Ed. Centauro. Caracas 1979.

EstelLAR, D.: Weekend en las guerrillas: memorias de un combatiente en dos épocas críticas de nuestra historia. Ed. Domingo Fuentes. Caracas 1983.

Garcia Pelayo, M.; Uslar Pietri, A., y otros: Seguridad y Defensa. Instituto Altos Estudios de la Defensa Nacional. IAEPEN, vol. I. Caracas.

Garcia Ponce, Guillermo: El Terrorismo. Ed. Ateneo. Caracas 1980.

GIL YePES, José: «Encaje político en el sector militar. El caso Venezuela». En Nueva Sociedad, n. ${ }^{\circ} 81$. Caracas 1986.

Gilmore, R. L.: Caudillismo and militarism in Venezuela, 1810-1910. Athens. Ohio 1964.

Grases, P., y Pérez Villa, M.: “Las Fuerzas Armadas de Venezuela», en Institución del siglo XX. Ed. Seix Barral. Caracas 1981.

JeCAMBI, O.; FeRnANDEZ, E., y otros: Seguridad y desarrollo. Instituto de Altos Estudios de la Defensa Nacional. Caracas 1979.

Martinez, Rafael: Aqui todo el mundo está alzao. Ojo de Camello. Caracas 1973.

MULler Rojas, Alberto: «Equipamiento militar, política de defensa y política exterior. El caso venezolano», en Política Internacional, n. ${ }^{\circ}$ 2. Caracas 1986.

-: «La idea de seguridad en los Estados Democráticos: Venezuela», en Politica Internacional, n. ${ }^{\circ} 8$. Caracas 1987.

PENA, Alfredo: Democracia y golpe militar. Ed. Ateneo. Caracas 1980.

-: Conversaciones con Douglas Bravo. Ed. Ateneo. Caracas 1979. 
PlazA, Elena: Historia de la lucha armada en Venezuela, 1960-69. Ed. Gunilla. Caracas.

RANGEL, D.: Seguridad, defensa y democracia. Un tema para civiles y militares. Ed. Centauro. Caracas 1980.

Rangel, J.: Militares y política. Ed. Centauro. Caracaș 1976.

-: «Problemas fundamentales de seguridad y defensa de Venezuela», en Seguridad, Defensa y Democracia. Ed. Aníbal Romero. 1980.

Rangel Bourgoin, L. E.: Nosotros los militares. Ed. Sol. Caracas 1983.

Rey, J. C.; Peñalara, C., y otros: “Dossier sobre Seguridad y Defensa. Democracia y participación política", en Estado y Reforma, n. ${ }^{\circ}$ 2. Caracas 1987 .

Rincon, Fredy: El nuevo ideal nacional y los planes económico-militares de Pérez Jiménez, 1952-1957. Ed. Centauro. Caracas 1982.

Rodriguez VALMORE: Bayonetas sobre Venezuela. Eds. Beatriz de Silva. México 1950.

RuIz PinedA, Leonardo: Guerrilleros de la libertad. Ed. Centauro. Caracas 1977.

SCHAPOSNIK, Eduardo: La democratización de las Fuerzas Armadas venezolanas. ILDIS. Caracas 1985.

SuCRE, L.: Gobernadores y Capitanes Generales de Venezuela. Ministerio de Relaciones Exteriores.

YEPEL DAZA, Jacobo: «El realismo militar venezolano», en El caso Venezuela, una ilusión de armonia. Ed. IESA. Caracas 1986.

-: Los fundamentos de la defensa de Venezuela. Instituto de Altos Estudios de la Defensa Nacional. Caracas 1980.

Zamora, Antonio: Memorias de la guerrilla venezolana. Síntesis dos mil. Caracas 1974.

ZiEMES, Ángel: El gomecismo y la formación del Ejército Nacional. Caracas 1979. 